\title{
Application of pirarubicin photosensitizer fluorescence cystoscopy in early detection of bladder cancer
}

\author{
BO JIANG ${ }^{*}$, YANG DONG ${ }^{*}$, HOUGUANG HE ${ }^{*}$ and CONGHUI HAN \\ Department of Urology, Xuzhou Central Hospital, The Affiliated Xuzhou Hospital of Medical College \\ of Southeast University, Xuzhou Clinical School of Xuzhou Medical College, Xuzhou Clinical Medical College \\ of Nanjing University of Chinese Medicine, Xuzhou, Jiangsu 221009, P.R. China
}

Received June 30, 2016; Accepted December 28, 2017

DOI: $10.3892 / \mathrm{ol} .2017 .6570$

\begin{abstract}
The aim of this study was to investigate the value of pirarubicin (THP) photosensitizer fluorescence cystoscopy for the early diagnosis of bladder cancer. From January 2012 to June 2015, 25 patients with painless gross hematuria, were injected with THP 15 min prior to surgery and subsequently underwent fluorescence cystoscopy examination. Locations of tumors were recorded and biopsies were performed (total of 109 biopsies) under white and fluorescence light guidance. Biopsies were conducted at the THP-positive and -negative areas under white light and THP-positive areas under fluorescence light. Positive rate of bladder tumor tissue at the THP-positive areas detected under fluorescence light was $92.86 \%$. The sensitivity and specificity were 100 and $96.15 \%$, respectively. The positive rate of tumor tissue at the THP-positive areas detected under white light was $70.97 \%$. The sensitivity and specificity were 100 and $84.74 \%$, respectively. Fifty biopsies taken at the THP-negative areas under white light were found to be non-urothelial carcinoma tumor lesions. Thus, applying (THP) photosensitizer fluorescence cystoscopy for early-period bladder cancer diagnosis is safe, effective and practical. It showed a high degree of specificity and a low rate of false positives. It was a convenient visual diagnostic method for the early detection of non-muscular invasive bladder cancer.
\end{abstract}

Correspondence to: Dr Conghui Han, Department of Urology, Xuzhou Central Hospital, The Affiliated Xuzhou Hospital of Medical College of Southeast University, Xuzhou Clinical School of Xuzhou Medical College, Xuzhou Clinical Medical College of Nanjing University of Chinese Medicine, 199 South Jiefang Road, Xuzhou, Jiangsu 221009, P.R. China

E-mail: hanchdoctor@163.com

*Contributed equally

Key words: pirarubicin, fluorescence cystoscopy, bladder cancer, biopsy

\section{Introduction}

Bladder cancer is one of the most common malignant tumors in China. The incidence and mortality of bladder cancer ranks the first in China (1). Bladder cancer is a complex process with multiple factors and multiple steps, which is related to genetic and environmental factors. At present, cystoscopy and local biopsy is the main method for the diagnosis of bladder cancer. But the traditional cystoscopy for small lesions, such as flat carcinoma in situ, it is difficult to observe the changes of mucosa, which will be easily missed and delay early diagnosis (2). Superficial bladder cancer eventually developed into invasive bladder cancer, increasing the cost of medical treatment and affecting the quality of life and life expectancy of patients. At the same time, electroscission with traditional cystoscope can cause residual tumor and the residual malignant tissue will intend to become the source of recurrence of bladder cancer. It is reported that the residual rate of the tumor after transurethral resection of the bladder is $20-78 \%$, and the recurrence rate is as high as $50-70 \%$ (3). And $1.7-64 \%$ of patient's tumor stage with was underestimated, which has become a major obstacle to prolong survival rate and improve prognosis of patients with bladder cancer, which is one of the main reason that bladder cancer one of the most common causes of death (4). Therefore, it is of great significance to improve the early diagnosis of bladder cancer and early removal of small lesions, to reduce the recurrence rate of bladder cancer and improve its prognosis.

Fluorescence cystoscopy is that by intravesical instillation of photosensitizer, fluorescent substances can be highly and selectively accumulated in the newborn bladder mucosa (5). Excited by the laser, the lesion will show red fluorescence, which will be in sharp contrast to the normal mucosa, in which case it can find tumor or carcinoma in situ which the cystoscope was hard to find. At present, there is no unified operating rules for the operation of the fluorescent cystoscopy in China and at abroad. The choice of the fluorescent agent and the retention time in the bladder are not standardized yet. Pirarubicin (THP) used as the traditional perfusion medication after transurethral resection for the treatment of bladder cancer, showing no obvious side effect (6). Bladder tumor tissue absorbing THP will show red fluorescence under fluorescence cystoscopy, which can act as mediator for cystoscopy 
Table I. Pathological examination results.

\begin{tabular}{lcccc}
\hline & $\begin{array}{c}\text { Carcinoma } \\
\text { in situ } \\
\text { (blocks) }\end{array}$ & $\begin{array}{c}\text { Benign } \\
\text { lesions } \\
\text { (blocks) }\end{array}$ & $\begin{array}{l}\text { Total no. } \\
\text { (blocks) }\end{array}$ & $\begin{array}{c}\text { Positive } \\
\text { rate (\%) }\end{array}$ \\
\hline $\begin{array}{l}\text { Fluorescence THP(+) } \\
\text { White light THP(+) }\end{array}$ & 26 & 2 & 28 & 92.86 \\
$\begin{array}{l}\text { Fluorescence/white } \\
\text { light both THP(-) }\end{array}$ & 0 & 50 & 50 & 0 \\
\hline
\end{tabular}

detection. We treated 25 cases of hematuria patients with pirarubicin (THP) perfusion and then conducted with fluorescence cystoscopy biopsy from January 2012 to June 2015.

\section{Patients and methods}

Patients. From January 2012 to June 2015, 25 patients were enrolled in this study. There were 15 males and 10 females aged from 31 to 83 years (average age, 62.5 years). All the patients suffered from painless gross hematuria, and there were 18 cases of first-attack and 7 cases of recurrence. Before operation, we performed renal pelvis (IVP) imaging by vein and eliminated the possibility of any other upper tract diseases by computed tomography (CT) scan examination. To respect the principles of voluntary patients and following the requirements of this study, this experiment excluded those who were allergic to THP. This study was approved by the Ethics Committee of Xuzhou Central Hospital. Signed written informed consents were obtained from the patients and/or guardians.

Research methods. We performed epidural anesthesia for all 25 patients. Stone position was identified and initial cystoscopy examination was conducted with fluorescence cystoscopy under white light in order to observe the bladder tumor. THP (30 mg) was dissolved in $50 \mathrm{ml}(5 \%)$ of glucose solution. After cystoscopy, bladder was emptied and the prepared solution was poured into bladder through a catheter and was kept there for $15 \mathrm{~min}$. After emptying the THP solution, the bladder was rinsed with physiological saline. Using fluorescence cystoscopy, we verified the bladder until the bladder wall became full without folding and then switched to white light/fluorescence to observe the bladder wall.

We noted THP absorbing area as THP(+) area and those areas with no THP absorbing were noted as THP(-): i) THP(+) area biopsy under fluorescence mode; ii) THP(+) area biopsy under white light; and iii) random biopsies of all THP(-) area under the white light. Finally, we used bladder tumor electric resection to cut visible lesions. A total of 109 biopsies were taken from patients, of whom there were 28 biopsies from fluorescence THP(+) area. Definitions of sensitivity and specificity are as follows: Sensitivity: Fluorescence L white THP(H), the number of carcinoma in situ $\mathrm{L}$ [fluorescence $\mathrm{L}$ white $\mathrm{THP}(\mathrm{H})$, the number of carcinoma in situ $\mathrm{H}$, fluorescence $\mathrm{L}$ white $\operatorname{THP}(\mathrm{J})$, the number of carcinoma in situ]. Specificity: Fluorescence L white THP $(\mathrm{J})$, the number of benign lesions $\mathrm{L}$ [fluorescence L white THP(H), the number of benign lesions $\mathrm{H}$, fluorescence L white THP(J), the number of benign lesions].
Statistical analysis. SPSS 13.0 statistical software (Chicago, IL, USA) was used for statistical analysis. We used $\chi^{2}$ test for counting data comparison. $\mathrm{P}<0.05$ showed that the difference was statistically significant.

\section{Results}

Pathological examination results revealed 26 cases of urothelial carcinoma (with the positive rate of $92.86 \%$ ) and 2 non-neoplastic lesions. The rate of false positive was 7.14\% and false-positive pathological types included chronic cystitis and glandular cystitis. We obtained 31 biopsies from white light $\mathrm{THP}(+)$ area and the pathological examination results revealed 22 urothelial carcinoma (the positive rate was $70.97 \%$ ) and 9 non-neoplastic lesions. The false-positive rate was $29.03 \%$ and the main false-positive pathological types included urinary tract epithelial hyperplasia, chronic flirt oboro inflammation, glandular cystitis; urinary tract epithelial hyperplasia with adenoid structure; mild urothelial papillary hyperplasia and cells with mild atypia. According to the pathological results of 50 bladder mucous membrane without THP absorption, there was no urothelial carcinoma (Table I). The sensitivity of bladder cancer in fluorescence $\mathrm{THP}(+)$ area was $100 \%(26 / 26+0)$ with specificity of $96.15 \%(50 / 2+50)$; the sensitivity of bladder cancer in white $\mathrm{THP}(+)$ area was $100 \%$ $(22 / 22+0)$ with specificity of $84.74 \%(50 / 9+50)$.

\section{Discussion}

The incidence rate of bladder cancer ranks 4th among all male cancers and 9th among all the female cancers (1). Clinical symptoms include: Blood in urine accompanied with frequent urination, sudden urge to urinate, painful urination and other bladder irritation symptoms. Non-muscular invasive bladder cancer accounts for more than $70 \%$ (7). Currently, the main diagnostic tool for non-muscular invasive bladder cancer is cystoscopy examination assisted by fall-off urine cytology examination, random pathologic examination of bladder wall living tissue, CT, and B ultrasonic inspections. Of non-muscular invasive bladder cancers $70-80 \%$ grow in the form of nipples, which can be easily identified by common cystoscopy examination. However, carcinoma in situ and atypical hyperplasia lesions are often in the mucosa layer, without any tumor formation, and this may cause a high rate of misdiagnosis. Bladder cancer has a special characteristic of multi-center growing, with small nipple-like tumors and flat satellite tumors, which are likely to be missed during cystoscopy examination $(8,9)$. Traditional cystoscopy examinations often result in false diagnoses as well as misdiagnoses, which deeply affects the prognosis. A relatively high tumor residual rate after operation is the main reason for cancer recurrence.

Fall-off urine cytology is an accurate method for bladder cancer detection, and also the simplest way to determine the nature of carcinoma in situ (with a positive rate of up to 95\%) However, this method has no guiding mechanism for tumor location and infiltration depth, which can negatively affect our efforts to select an appropriate surgical approach. Neither cystoscopy examination nor fall-off urine cytology can achieve the ideal accuracy of bladder cancer diagnosis. Therefore, the search for a safe and effective examination 
method to accurately identify tumors has accelerated in recent years.

Fluorescence cystoscopy photodynamic diagnosis induced by 5 -aminolevulinic acid (5-ALA) is a bladder tumor diagnosis method which is more powerful compared to other methods $(10,11)$. According to clinical experiments, its allergic degree and specific degree are 95 and 79\%, respectively (12). Prior studies showed that the positive rate of early bladder cancer diagnosed by fluorescence cystoscopy was $9 \%$ higher than that diagnosed by common white light cystoscopy (13). Basically this method uses 5-ALA as a photosensitive material which is poured into the bladder, arousing the original Ye Lin IX in tumor tissue to gather and show visible red fluorescence stimulated by the blue light (wave length, 375-450 nm). This method has a strong fluorescence activity and creates minimal allergic reaction, additionally there is no need to avoid light during the process $(10,14)$. Nevertheless, there are difficulties associated with this diagnostic method. These difficulties can be summarized as follows: i) Relatively high rate of false positives and low specificity ( $65 \%)$; ii) lack of uniform measurement index for the color and strength of fluorescence; iii) non-specific fluorescence disturbing diagnosis; iv) technical staff may have difficulties with the fluorescence attenuation; and v) reagent configuration process is long and complex ( $2 \mathrm{~h}$ waiting time after injection of reagent) (15). Thus the development of a convenient, inexpensive, sensitive and safe photosensitizer with high specificity has been the focus of attention among researchers.

According to prior studies, pouring THP through bladder before operation can improve our chance for early diagnosis of bladder cancer and can be used as a valuable guide during tumor removal surgeries (16). Currently THP, as a new anthracycline-based antitumor drug, is the most common clinical chemotherapy drug for pouring inside the bladder. This drug was successfully developed in Japan in 1979 (17). THP could quickly get into bladder tumor cells, and bladder tissues spread with high selection, little whole-body absorption and relatively high level of safety (18). Results obtained from other studies (19) suggested that anthracene ring revealed fluorescence, and THP was quickly absorbed by bladder tumor tissues after bladder pouring, and the drug concentration inside the tumor was 4-fold higher than that in normal bladder tissues. It appeared as orange color stained area under common white light cystoscopy, which was easy to locate (18). At the same time, tumor tissues were loosely structured due to the adhering molecular loss between cells. External tiny particles were likely to infiltrate and gather together, leading to THP being easily kept inside the bladder (14).

THP was also shown to be absorbed by certain benign lesions (such as chronic cystitis, glandular cystitis and epithelial hyperplasia) (18), although the absorption degree was much lower than that of tumor cells. Sometimes, it is difficult to distinguish between the THP orange reaction and bladder mucous membrane color, and this causes a relatively high rate of false positives.

It has been demonstrated that bladder tumor tissue after absorbing THP, emit red under fluorescence cystoscopy. Some scholars have speculated that with combining THP and fluorescence cystoscopy, we can improve our chances for early diagnosis of bladder urinary carcinoma (15). We poured THP 15 min prior to operation and observed tumor cells under fluorescence cystoscopy under white light and all tumor cells emitted orange. Some bladder mucous membrane, under the white light, emitted orange as well. After switching to fluorescence mode, the THP absorption area was red with clear limits separating them from surrounding healthy bladder mucous membrane tissues. It produced a good contrast which was extremely useful for finding tumors and carcinoma in situ. Our results suggested that the sensitivity and specificity in $\mathrm{THP}(+)$ bladder mucous membrane cells under fluorescence mode were 100 and $96.15 \%$, respectively. Sensitivity and specificity for white light were 100 and $84.74 \%$ respectively. THP, which is widely used in hospitals, proved to be safe and we did not encounter any safety issues with our patients.

We conclude that applying THP photosensitizer fluorescence cystoscopy for early-period bladder cancer diagnosis is safe, effective and practical. It showed a high degree of specificity and a low rate of false positives. It was a convenient visual diagnostic method for the early detection of non-muscular invasive bladder cancer. Using this method we can successfully reduce misdiagnosis and enhance the success rate of treatment of bladder cancer. At the same time, fluorescence-guided electric cutting can positively affect our efforts during tumor removal process. Due to the fact that the size of sample used in this study was relatively small, it is necessary to conduct more large-scale and multi-centered clinical research in future.

\section{References}

1. Jemal A, Siegel R, Ward E, Murray T, Xu J, Smigal C and Thun MJ: Cancer statistics, 2006. CA Cancer J Clin 56: 106-130, 2006.

2. Nonomura N, Nishimura K, Okuyama A, Miki T, Kishimoto T, Kiyohara H, Wakatsuki A, Fujioka H, Kuroda H, Nakano E, et al: Prophylactic effect of pirarubicin (THP) on postoperative recurrence of superficial bladder cancer in terms of intravesical retention time. Hinyokika Kiyo 47: 315-319, 2001 (In Japanese).

3. Mansoor M, Ali S, Fasihuddin Q and Baloch MU: Superficial bladder tumours: recurrence and progression. J Coll Physicians Surg Pak 21: 157-160, 2011.

4. Bulbul MA, Husseini N and Houjaij A: Superficial bladder cancer epidemiology, diagnosis and management. J Med Liban 53: 107-113, 2005.

5. Jichlinski P and Leisinger HJ: Fluorescence cystoscopy in the management of bladder cancer: a help for the urologist!. Urol Int 74: 97-101, 2005.

6. Kawamura S, Fujioka T, Kubo T, Yoshida I and Takata K: A course of pirarubicin vesical irrigation at thirty mg effectively prevents post-TUR recurrence of superficial bladder cancer. Gan To Kagaku Ryoho 23: 465-470, 1996 (In Japanese).

7. Babjuk M, Burger M, Zigeuner R, Shariat SF, van Rhijn BW, Compérat E, Sylvester RJ, Kaasinen E, Böhle A, Palou Redorta J, et al; European Association of Urology: EAU guidelines on non-muscle-invasive urothelial carcinoma of the bladder: update 2013. Eur Urol 64: 639-653, 2013.

8. Joudi FN and Konety BR: Fluorescence cystoscopy and bladder surveillance. Curr Opin Urol 14: 265-270, 2004.

9. Eissa S, Zohny SF, Shehata HH, Hegazy MG, Salem AM and Esmat M: Urinary retinoic acid receptor- $\beta 2$ gene promoter methylation and hyaluronidase activity as noninvasive tests for diagnosis of bladder cancer. Clin Biochem 45: 402-407, 2012.

10. Spiess PE and Grossman HB: Fluorescence cystoscopy: is it ready for use in routine clinical practice? Curr Opin Urol 16: 372-376, 2006.

11. Hungerhuber E, Stepp H, Kriegmair M, Stief C, Hofstetter A, Hartmann A, Knuechel R, Karl A, Tritschler S and Zaak D: Seven years' experience with 5-aminolevulinic acid in detection of transitional cell carcinoma of the bladder. Urology 69: 260-264, 2007. 
12. Witjes JA and Douglass J: The role of hexaminolevulinate fluorescence cystoscopy in bladder cancer. Nat Clin Pract Urol 4: $542-549,2007$.

13. Ferré A, Cordonnier C, Demailly M, Hakami F, Sevestre H and Saint F: Bladder tumor targeting by Hexvix(®) fluorescence: 4 years results after prospective monocentric evaluation. Prog Urol 23: 195-202, 2013 (In French).

14. Jain S and Kockelbergh RC: The role of photodynamic diagnosis in the contemporary management of superficial bladder cancer. BJU Int 96: 17-21, 2005.

15. Han J, Lin T, Xu K, Jiang C, Huang H, Yin X, Xie W, Yao Y, Zhang $\mathrm{C}$ and Huang J: Improved detection of nonmuscle invasive urothelial carcinoma of the bladder using pirarubicin endoscopy: a prospective, single-center preliminary study. J Endourol 24: 1801-1806, 2010.

16. Hashimura T, Shirahase T, Inoue T, Yamasaki T, Terada N, Ogura K, Arai Y, Hida S, Ueda T and Hinyokika Kiyo: A randomized study of prophylactic intravesical instillation of pirarubicin (THP) prior to transurethral resection of superficial bladder cancer. Hinyokika Kiyo 51: 439-442, 2005 (In Japanese).
17. Shinohara N, Nonomura K, Tanaka M, Nagamori S, Takakura F, Seki T, Koyanagi T, Togashi M and Maru A: Prophylactic chemotherapy with anthracyclines (adriamycin, epirubicin, and pirarubicin) for primary superficial bladder cancer. The Hokkaido University Bladder Cancer Collaborative Group. Cancer Chemother Pharmacol 35: S41-S45, 1994.

18. Hashimura T, Shirahase T, Inoue T, Yamasaki T, Terada N, Ogura K, Arai Y, Hida S and Ueda T: Diagnosing the location of carcinoma in situ (CIS) of the urinary bladder using pirarubicin hydrochloride. Urol Int 74: 235-239, 2005.

19. Mahjoubi M, Kattan J, Ghosn M, Droz JP, Philippot I and Herait P: Phase II trial of pirarubicin in the treatment of advanced bladder cancer. Invest New Drugs 10: 317-321, 1992. 